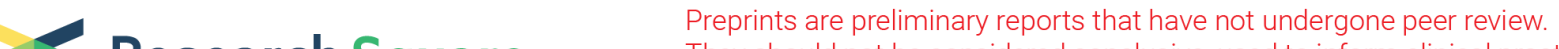 Research Square They should not be considered conclusive, used to inform clinical practice, or referenced by the media as validated information.
}

\section{hMAGEA2 Promotes Cell Growth and Tumorigenicity in Melanoma}

\section{Junkoo Yi}

Gyeongbuk Livestock Research Institute

\section{Song Park}

DGIST: Daegu Gyeongbuk Institute of Science and Technology

\section{Hai Huang}

kyungpook national University

Haibo Zhang

kyungpook National University

\section{Eun Gyung Kim}

Kyungpook National University

\section{Kang Dong Liu}

The China-US(Henan) Hormel Cancer Institute

\section{Seong Kyoon Choi}

DGIST

\section{Zae Young Ryoo}

Kyungpook national University

\section{Myoung-Ok Kim ( $\sim$ 79lee38@korea.kr)}

Kyungpook National University https://orcid.org/0000-0001-6650-7734

\section{Primary research}

Keywords: Melanoma, MAGEA, SK-MEL-5, SK-MEL-28

Posted Date: October 28th, 2020

DOI: https://doi.org/10.21203/rs.3.rs-95048/v1

License: (c) (1) This work is licensed under a Creative Commons Attribution 4.0 International License. Read Full License 


\section{Abstract}

Background: Melanoma is one of the most malignant skin cancers, and its incidence continues to rise worldwide, especially in fair-skinned populations. A clinical challenge with melanoma is that it has no unique or specific clinical presentation. The clinical presentation of melanoma varies with the anatomic localization, type of growth, and histopathology. Proteins belonging to the melanoma-associated antigen (MAGEA) gene family are typically expressed in germline cells but differentially expressed in a variety of human cancers, particularly melanoma. Melanoma-associated antigen 2 (MAGEA2) is such a protein. In this study, we investigated whether the expression of human MAGEA2 (hMAGEA2) is associated with melanoma.

Methods: SK-MEL-5 and SK-MEL-28 cell lines were used to explore the cellular and molecular mechanisms underlying the progression and invasiveness of melanoma. A melanoma human tissue array was used to examine the correlation between the progression of melanoma and expression of hMAGEA2

Results: hMAGEA2 is overexpressed in human melanoma tissues, including metastatic tissues. In SKMEL-5 and SK-MEL-28 cells, overexpression of hMAGEA2 increased cellular proliferation and colony formation, whereas hMAEGA2 knockdown suppressed cellular proliferation, colony formation, and migration.

Conclusion: These results show that the hMAGEA2 protein plays a role in the growth and invasiveness of melanoma cells, and it is correlated with melanoma metastasis. Therefore, hMAGEA2 contributes to the progression of melanoma and may be a diagnostic and novel therapeutic target for the treatment of melanoma metastasis patients.

\section{Background}

Malignant melanoma, a skin cancer that arises from the malignant transformation of melanocytes, is highly aggressive and metastatic [1]. Patient prognosis is typically poor, and the 5-year survival rate is < $15 \%[2,3]$. Our understanding of melanoma pathobiology and genetics has increased in recent years; consequently, clinical treatments are more effective. Such treatments include targeted therapies and immunotherapies, both of which contribute to the regression of advanced disease; however, few are curative in many patients [4]. The complicated molecular mechanism of malignant melanoma development and progression requires continued study.

Melanoma-associated antigens (MAGEs) are members of a gene family that consists of $>50$ proteins. MAGEs are divided into two types, MAGE-I and MAGE-II, according to gene structure and tissue-specific expression patterns $[5,6]$. The MAGE-I family members (collectively called MAGE hereafter) are encoded by 28 genes clustered on the $X$ chromosome, including MAGE-A, - $b$ and $-C$, which are typically expressed in cancer cells of various origins, but not in adult tissues, except for germline cells in the placenta, ovaries, and testes [7]. By contrast, the MAGE-II subgroup, including MAGE-D variants, do not have defined 
chromosome clustering, is not cancer-specific, and is ubiquitously expressed in normal adult tissues and germline cells [6-8]. MAGE proteins act as anti-tumoral immune targets, making these antigens a focus of immunotherapy research in gastrointestinal carcinoma and other cancers [5]. MAGE expression is an independent prognostic variable for metastasis and the extent of relapse-free survival, comparable to Breslow thickness and ulceration in melanoma [9]. However, despite intensive study, the molecular mechanisms of hMAGEA2 in the development and progression of melanoma remain poorly understood.

In this study, SK-MEL-5 and SK-MEL-28 cell lines were used to explore the cellular and molecular mechanisms underlying the progression and invasiveness of melanoma. A melanoma human tissue array was used to examine the correlation between the progression of melanoma and expression of hMAGEA2. We found that hMAGEA2 expression was significantly increased in melanoma tumors, including metastatic tumors. In vitro studies reveals that in SK-MEL-5 and SK-MEL-28 cell lines that overexpress hMAGEA2, cellular proliferation and colony formation were significantly increased. Conversely, knockdown of hMAGEA2 expression inhibited melanoma cell proliferation and migration. These results suggest that hMAGEA2 plays a role in the growth and invasiveness of melanoma cells and that its overexpression is correlated with melanoma metastasis. Therefore, hMAGEA2 may contribute to the progression of melanoma in vivo and is a potential diagnostic and novel therapeutic target for the treatment of melanoma patients.

\section{Methods}

\section{Construction of tissue microarray and immunohistochemistry}

The tissue microarrays (TMAs) were constructed with $1 \mathrm{~mm}$ diameter cores punched from two or three distinct regions from each formalin-fixed paraffin-embedded tumor blocks. The TMAs were assembled with a tissue arrayer (Beecher Instruments, Silver Spring, MD). One section from each TMA was stained with hematoxylin and eosin and reviewed to confirm the presence of representative tumors. After deparaffinization, rehydration, and antigen retrieval, the hMAGEA2 protein was detected by immunohistochemistry using antibodies (sc-130164, Santa Cruz) and the Ultraview Universal DAB Detection Kit (Ventana, Tucson, AZ). All sections were counterstained with hematoxylin. TMA sections were assessed for the intensity of the stain and the percentage of cells with staining in the nucleus, cytoplasm, and cell membrane. Staining was considered positive when there moderate or strong immune reactivity at the appropriate location over the cut-off point. A sample of 45 cases of malignant melanoma tissues, 20 normal skin tissues, and 10 adjacent cancer skin tissues.

\section{Cell culture and transfection}

Media components for cell culture were purchased from Thermo Fisher Scientific (Grand Island, NY, USA). The human melanoma cell lines SK-MEL- 5 and SK-MEL-28 were cultured in minimum essential medium (MEM) supplemented with $10 \%$ fetal bovine serum (FBS) and 1\% penicillin/streptomycin (P/S). For stable 
expression of hMAGEA2 in melanoma cell lines, cells were seeded into $100 \mathrm{~mm}$ dishes. After $24 \mathrm{~h}$, cells were transfected with pcDNA3.1-mock or pcDNA3.1-hMAGEA2 vector using FuGENE HD (Promega, Madison, $\mathrm{WI}$ ) transfection reagent following the manufacturer's instructions. To establish stable expression, the transfected melanoma cell lines were treated with $\mathrm{G} 418(1.5 \mathrm{mg} / \mathrm{ml})$ for 7 days. Subcultures were treated with G418 every 3 days. All cell lines were in an incubator maintained at $37^{\circ} \mathrm{C}$ with $5 \% \mathrm{CO}_{2}$.

\section{Cell proliferation (CCK-8) assay}

Cell proliferation was estimated using the CCK-8 assay (Cell Counting Kit-8, Dojindo Molecular Technologies, Inc.). Cells were seeded into 96 -well plates $\left(1 \times 10^{3}\right.$ cells per well $)$ and incubated for 0,24 , 48,72 , and $96 \mathrm{~h}$. In total, $10 \mu \mathrm{l}$ of CCK-8 solution was added to each well and incubated for an additional $1 \mathrm{~h}$ at $37^{\circ} \mathrm{C}$. The optical density (OD) of each well was measured at $450 \mathrm{~nm}$ using a spectrophotometer (BioTek).

\section{Anchorage-independent colony formation assay}

The effects of hMAGEA2 on anchorage-independent growth were investigated in the melanoma cell lines. Cells $\left(8 \times 10^{3}\right.$ cells per $\left.\mathrm{ml}\right)$, suspended in MEM supplemented with $10 \% \mathrm{FBS}$ and $1 \% \mathrm{P} / \mathrm{S}$, were added to the top layer of $0.3 \%$ agar, over a base layer of $0.5 \%$ agar. The cultures were maintained at $37{ }^{\circ} \mathrm{C}$ in a $5 \%$ $\mathrm{CO}_{2}$ incubator for 1-2 weeks and then colonies were counted under a microscope (Leica) using ImagePro Plus software (V.4)

\section{Wound healing assay}

Wound healing assays were performed to evaluate cell motility. Melanoma cells were cultured in 6-well dishes at $1 \times 10^{6}$ cells/well. A single scratch wound was established by scraping a p10 micropipette tip across the surface of a plate of confluent cells. Cells were washed with PBS three times to remove cell debris, and the assay medium was added. Images of the scratched area of each plate were obtained by light microscopy (Leica) at 10X magnification at 0,12 , and $24 \mathrm{~h}$, and the distance of the gaps was evaluated.

\section{Cell cycle analysis}

Cells $\left(7 \times 10^{4}\right)$ were seeded in a $60 \mathrm{~mm}$ dish and cultured for $48 \mathrm{~h}$. The cells were harvested by trypsinization, fixed with ethanol, stained with propidium iodide, and then analyzed for cell cycle phase by flow cytometry.

\section{Lentiviral production and infection}

The lentiviral hMAGEA2 shRNA vectors (sh-hMAGEA2 sequence: 5'-GATAATCGTCCTGGCCATAAT-3') for the knockdown of hMAGEA2 were purchased from Sigma. Oligonucleotides were cloned into the pLKO.1 lentiviral vector. HEK293T cells were co-transfected with pLK0.1-scramble or pLKO.1-shMAGEA2 and pMDLg/p RRE, pMD2.G, and pRSV-Rev using FuGENE HD transfection reagent (Promega). SK-MEL-5 and SK-MEL-28 cells were infected with lentiviruses encoding shRNA using $8 \mu \mathrm{g} / \mathrm{ml}$ protamine sulfate 
(Sigma). After $48 \mathrm{~h}$, cells were selected by puromycin $(1 \mu \mathrm{g} / \mathrm{ml})$ for 4 days to establish a stable knockdown of hMAGEA2 in the SK-MEL-5 and SK-MEL-28 cell lines.

\section{Xenograft mouse model}

The experimental protocols were approved by the Animal Care and Use Committee of Kyungbook National University. Athymic nude mice (6-week-old; mean body weight $20 \mathrm{~g}$ ) were obtained from Orient (Seoul, Korea). Animals were acclimated to the facility for 1 week before the study and had free access to food and water. Tumor volume was calculated from two measurements of the diameter of the tumor base using the formula: tumor volume $\left(\mathrm{mm}^{3}\right)=($ length $\times$ width $\times$ height $\times 0.5)$. Mice were monitored until tumors reached $1 \mathrm{~cm}^{3}$ in total volume and were euthanized for further studies.

\section{Statistical analysis}

Data are presented as mean \pm SEM. All in vitro experiments were performed in triplicate. One-way analysis of variance (ANOVA) with Tukey's post hoc analysis was used to evaluate statistical differences between groups. Statistical analyses were carried out using GraphPad Prism 5 software (GraphPad Software Inc., $\mathrm{CA}$ ). The correlation between the two parameters was determined by the Spearman correlation and kappa coefficient. $P \leq 0.05$ was considered statistically significant.

\section{Results}

\section{hMAGEA2 is highly expressed in human melanoma tissues}

hMAGEA2 is overexpressed in a variety of human cancers, and its overexpression was found to be correlated with a worse prognosis [5, 10-13]. To investigate whether hMAGEA2 is overexpressed in human melanoma tissues and whether MAGE expression increases with metastasis, we measured expression levels of hMAGEA2 in human melanoma tissues using TMAs. Results showed that the expression level of hMAGEA2 was significantly upregulated in human melanoma cancer tissues compared with adjacent cancer tissues and normal tissues $(P<0.01)$ (Figure 1A, 1B), and hMAGEA2 expression was significantly higher in melanoma with metastasis than in melanoma without metastasis $(P<0.01)$ (Figure 1C). These findings suggest that hMAGEA2 is associated with melanoma metastasis and tumorigenesis.

\section{Overexpression of hMAGEA2 in melanoma cell lines promotes cell growth.}

To investigate the functional relevance of hMAGEA2 in melanoma, we established SK-MEL-5 and SKMEL-28 cell lines that overexpress hMAGEA2 by stable transfection using a plasmid vector not expressing hMAGEA2 (mock vector) or a vector that drives the expression of hMAGEA2. Immunoblot analysis results showed that the expression of hMAGEA2 protein was increased significantly in SK-MEL-5 and SK-MEL-28 cell lines compared with mock-transfected cell lines (Figure2A). Cell proliferation was measured by the CCK-8 proliferation assay. Proliferation was measured at $0,24,48,72$, and $96 \mathrm{~h}$ in the stably transfected SK-MEL-5 and SK-MEL-28 cell lines. The results showed that the proliferation was increased in cell lines that overexpress hMAGEA2 relative to control cells (Figure2B). Our results 
demonstrate that the overexpression of hMAGEA2 in SK-MEL-5 and SK-MEL-28 increases cell proliferation.

\section{Knockdown of hMAGEA2 in melanoma cells suppresses cell growth}

To investigate the effects of hMAGEA2 downregulation, we established cells in which the overexpression of hMAGEA2 was suppressed in the stably transfected SK-MEL-5 and SK-MEL-28 cell lines using lentivirus infection. Immunoblot analysis of sh-hMAGEA2-, and sh-MOCK-infected melanoma cells revealed that expression of endogenous hMAGEA2 was suppressed relative to cells expressing sh-MOCK (Figure $3 \mathrm{~A}$ ). The results of the CCK-8 proliferation assays indicate that knockdown of hMAGEA2 significantly decreases the proliferation of SK-MEL-5 and SK-MEL-28 cells (Figure 3B). Overall, this data demonstrates that hMAGEA2 protein plays an important role in melanoma cell proliferation.

\section{hMAGEA2 inhibits the migration of melanoma cells}

Clinical pathology data analysis indicates that hMAGEA2 is involved in tumor metastasis. To confirm whether hMAGEA2 plays a role in the migration of SK-MEL-5 and SK-MEL-28cells, a wound-healing assay was performed. First, we constructed hMAGEA2 knockdown SK-MEL- 5 and 28 cells, and then measured the serum-induced migration of metastatic SK-MEL-5 and 28 cells. sh-hMAGEA2 decreased seruminduced cancer cell migration as measured by wound-healing assays (Figure 4A). Compared to SK-MEL-5 and 28 sh-MOCK cells, SK-MEL-5 and 28 sh-hMAGEA2 cell migration is significantly increased (Figure $4 \mathrm{~B})$. These results suggest that hMAGEA2 plays a key role in melanoma metastasis.

\section{hMAGEA2 is involved in the cell cycle of melanoma cells}

The effect of hMAGEA2 on cell cycle progression was analyzed by flow cytometry. The results suggest that overexpression hMAGEA2 induces G1 phase distribution arrest in SK-MEL-28 cells (Figure 5A) and that the knockdown of hMAGEA2 decreases G1 S phase in SK-MEL-5 cells (Figure 5B). These findings indicate that hMAGEA2 is associated with cell cycle characteristics.

\section{Knockdown of hMAGEA2 suppresses colony formation of melanoma cells in soft-agar}

The effect of suppressing hMAGEA2 expression on the ability of melanoma cells to form colonies in softagar was measured. Colony formation is decreased in sh-hMAGEA2 melanoma cells relative to sh-MOCK control cells (Figure 6A). In SK-MEL-5 and SK-MEL-28 cell lines, the number of colonies formed is significantly decreased by knockdown of hMAGEA2 (Figure 6B). Our results demonstrate that the knockdown of hMAGEA2 suppresses colony formation in soft-agar. Overall, the data demonstrate that the hMAGEA2 protein plays a key role in the anchorage independent growth of melanoma cells.

\section{hMAGEA2 enhances xenograft tumor formation}

To investigate the growth rate in vivo, we performed a xenograft tumor formation assay. The volume and diameter of tumors derived from SK-MEL-5 cell lines that overexpress hMAGEA2 were significantly 
increased relative to cells that do not overexpress hMAGEA2 (Figure 7).

\section{Discussion}

MAGEA proteins are known to be highly expressed in a wide range of cancers, including bladder, lung, ovary, melanoma, pancreas, and breast malignancies [5, 10-13]. Studies have focused primarily on their use as immunotherapeutic targets in cancer for many years. Because of high sequence homology among the MAGE gene family members, the proteins were considered functionally redundant. Recently, evidence suggests that individual MAGE proteins function in specific pathways critical for tumor progression [1419]. The expression of MAGEA3 stimulates cell cycle progression, rate of migration, and invasion in thyroid cells in vitro, and these characteristics are associated with aggressive behavior in tumors [20]. Silencing MAGEB in murine melanoma cells suppressed the growth of melanoma in vivo [21]. Recent reports propose that MAGEA2 silences downstream targets of p53 activation via the MAGEA2-p53 complex in melanoma [22]. MAGEA proteins can also activate specific RING-type ubiquitin E3 ligases [15, 23], thereby affecting ubiquitin signaling in cancer cells. MAGEA2 is overexpressed in glioma and is associated with poor prognosis in glioma patients [24]. Suppression of MAGEA2 in lung cancer cells significantly reduced the growth rate and survival of cancer cells [25]. hMAGEA2 promotes the progression of breast cancer by regulating Akt and Erk1/2 pathways [26]. Although recent studies have sought to understand the role of MAGE proteins in cancer, the cellular and physiological functions of MAGE proteins have not been fully elucidated.

The work presented here showed that hMAGEA2 overexpression increased the viability of melanoma cells in vitro and enhanced xenograft tumor formation in mice. We found that hMAGEA2 is significantly overexpressed in melanoma tissues. In SK-MEL-5 and SK-MEL-28 cell lines that overexpress hMAGEA2, cellular proliferation and colony formation was significantly increased. Conversely, knockdown of overexpressed hMAEGA2 in these SK-MEL-5 and SK-MEL-28 cell lines suppressed cellular proliferation, colony formation, and migration. Our results suggest that hMAGEA2 may play a role in melanoma metastasis and that hMAGEA2 is a potential therapeutic target in metastatic melanoma patients.

\section{Conclusion}

These results show that the hMAGEA2 protein plays a role in the growth and invasiveness of melanoma cells, and it is correlated with melanoma metastasis. Therefore, hMAGEA2 contributes to the progression of melanoma and may be a diagnostic and novel therapeutic target for the treatment of melanoma metastasis patients.

\section{List Of Abbreviations}

MAGEA: melanoma-associated antigen

MAGEA2: melanoma-associated antigen 2 
hMAGEA2: human melanoma-associated antigen 2

MAGEs: melanoma-associated antigens

TMAs: tissue microarrays

MEM: minimum essential medium

FBS: fetal bovine serum

P/S: penicillin/streptomycin

CCK-8: Cell Counting Kit-8

\section{Declarations}

\section{Ethics approval and consent to participate}

The animal experiments were conducted in accordance with the guidelines of the Institutional Animal Care and Use Committee of Kyungpook National University (KP-118).

\section{Consent for publication}

Not applicable.

\section{Availability of data and materials}

All data generated or analysed during this study are included in this published article.

\section{Competing interests}

The authors declare that they have no competing interests.

\section{Funding}

The study was performed without external funding

\section{Authors' contributions}

JK performed the majority of experiments, analyzed the data, and wrote the manuscript; Song, EG, KD, SK participated in animal experiments; Hai and Haibo assisted with data analysis and edited the manuscript, $\mathrm{ZY}$ designed and $\mathrm{MO}$ coordinated the research. All authors had read and approved the manuscript, and ensure that this is the case.

\section{Acknowledgements}

Not applicable. 


\section{References}

1. Miller AJ, Mihm MC, Jr.: Melanoma. N Engl J Med 2006, 355(1):51-65.

2. Pacheco I, Buzea C, Tron V: Towards new therapeutic approaches for malignant melanoma. Expert Rev Mol Med 2011, 13:e33.

3. Lujambio A, Lowe SW: The microcosmos of cancer. Nature 2012, 482(7385):347-355.

4. Shah DJ, Dronca RS: Latest advances in chemotherapeutic, targeted, and immune approaches in the treatment of metastatic melanoma. Mayo Clin Proc 2014, 89(4):504-519.

5. Chomez P, De Backer O, Bertrand M, De Plaen E, Boon T, Lucas S: An overview of the MAGE gene family with the identification of all human members of the family. Cancer Res 2001, 61(14):55445551.

6. Tseng HY, Chen LH, Ye Y, Tay KH, Jiang CC, Guo ST, Jin L, Hersey P, Zhang XD: The melanomaassociated antigen MAGE-D2 suppresses TRAIL receptor 2 and protects against TRAIL-induced apoptosis in human melanoma cells. Carcinogenesis 2012, 33(10):1871-1881.

7. Sang M, Wang L, Ding C, Zhou X, Wang B, Wang L, Lian Y, Shan B: Melanoma-associated antigen genes - an update. Cancer Lett 2011, 302(2):85-90.

8. Oya H, Kanda M, Takami H, Hibino S, Shimizu D, Niwa Y, Koike M, Nomoto S, Yamada S, Nishikawa Y et al: Overexpression of melanoma-associated antigen D4 is an independent prognostic factor in squamous cell carcinoma of the esophagus. Dis Esophagus 2015, 28(2):188-195.

9. Svobodova S, Browning J, MacGregor D, Pollara G, Scolyer RA, Murali R, Thompson JF, Deb S, Azad A, Davis ID et al: Cancer-testis antigen expression in primary cutaneous melanoma has independent prognostic value comparable to that of Breslow thickness, ulceration and mitotic rate. Eur J Cancer 2011, 47(3):460-469.

10. Brasseur F, Rimoldi D, Lienard D, Lethe B, Carrel S, Arienti F, Suter L, Vanwijck R, Bourlond A, Humblet $Y$ et al: Expression of MAGE genes in primary and metastatic cutaneous melanoma. Int $J$ Cancer 1995, 63(3):375-380.

11. Bergeron A, Picard V, LaRue H, Harel F, Hovington $H$, Lacombe L, Fradet Y: High frequency of MAGEA4 and MAGE-A9 expression in high-risk bladder cancer. Int J Cancer 2009, 125(6):1365-1371.

12. Schultz-Thater E, Piscuoglio S, lezzi G, Le Magnen C, Zajac P, Carafa V, Terracciano L, Tornillo L, Spagnoli GC: MAGE-A10 is a nuclear protein frequently expressed in high percentages of tumor cells in lung, skin and urothelial malignancies. Int J Cancer 2011, 129(5):1137-1148.

13. Hou S, Sang M, Zhao L, Hou R, Shan B: The expression of MAGE-C1 and MAGE-C2 in breast cancer and their clinical significance. Am J Surg 2016, 211(1):142-151.

14. Minges JT, Grossman G, Zhang P, Kafri T, Wilson EM: Post-translational Down-regulation of Melanoma Antigen-A11 (MAGE-A11) by Human p14-ARF Tumor Suppressor. J Biol Chem 2015, 290(41):25174-25187.

15. Gao Y, Mutter-Rottmayer E, Greenwalt AM, Goldfarb D, Yan F, Yang Y, Martinez-Chacin RC, Pearce KH, Tateishi S, Major MB et al: A neomorphic cancer cell-specific role of MAGE-A4 in trans-lesion 
synthesis. Nat Commun 2016, 7:12105.

16. Jiang J, Xie D, Zhang W, Xiao G, Wen J: Fusion of Hsp70 to Mage-a1 enhances the potency of vaccine-specific immune responses. $J$ Trans/ Med 2013, 11:300.

17. Chen X, Wang L, Liu J, Huang L, Yang L, Gao Q, Shi X, Li J, Li F, Zhang Z et al: Expression and prognostic relevance of MAGE-A3 and MAGE-C2 in non-small cell lung cancer. Oncol Lett 2017, 13(3):1609-1618.

18. Vujanovic L, Shi J, Kirkwood JM, Storkus WJ, Butterfield LH: Molecular mimicry of MAGE-A6 and Mycoplasma penetrans HF-2 epitopes in the induction of antitumor CD8(+) T-cell responses. Oncoimmunology 2014, 3(8):e954501.

19. Zhai X, Xu L, Zhang S, Zhu H, Mao G, Huang J: High expression levels of MAGE-A9 are correlated with unfavorable survival in lung adenocarcinoma. Oncotarget 2016, 7(4):4871-4881.

20. Liu W, Cheng S, Asa SL, Ezzat S: The melanoma-associated antigen A3 mediates fibronectincontrolled cancer progression and metastasis. Cancer Res 2008, 68(19):8104-8112.

21. Yang B, O'Herrin SM, Wu J, Reagan-Shaw S, Ma Y, Bhat KM, Gravekamp C, Setaluri V, Peters N, Hoffmann FM et al: MAGE-A, mMage-b, and MAGE-C proteins form complexes with KAP1 and suppress p53-dependent apoptosis in MAGE-positive cell lines. Cancer Res 2007, 67(20):9954-9962.

22. Monte M, Simonatto M, Peche LY, Bublik DR, Gobessi S, Pierotti MA, Rodolfo M, Schneider C: MAGE-A tumor antigens target p53 transactivation function through histone deacetylase recruitment and confer resistance to chemotherapeutic agents. Proc Natl Acad Sci U S A 2006, 103(30):11160-11165.

23. Doyle JM, Gao J, Wang J, Yang M, Potts PR: MAGE-RING protein complexes comprise a family of E3 ubiquitin ligases. Mol Cell 2010, 39(6):963-974.

24. Meng Q, Luo G, Liu B, Sun Y, Yan Z: Melanoma-associated antigen A2 is overexpressed in glioma and associated with poor prognosis in glioma patients. Neoplasma 2018, 65(4):604-609.

25. Ujiie H, Kato T, Lee D, Hu HP, Fujino K, Kaji M, Kaga K, Matsui Y, Yasufuku K: Overexpression of MAGEA2 has a prognostic significance and is a potential therapeutic target for patients with lung cancer. Int J Oncol 2017, 50(6):2154-2170.

26. Park S, Sung Y, Jeong J, Choi M, Lee J, Kwon W, Jang S, Park SJ, Kim HS, Lee MH et al: hMAGEA2 promotes progression of breast cancer by regulating Akt and Erk1/2 pathways. Oncotarget 2017, 8(23):37115-37127.

\section{Figures}



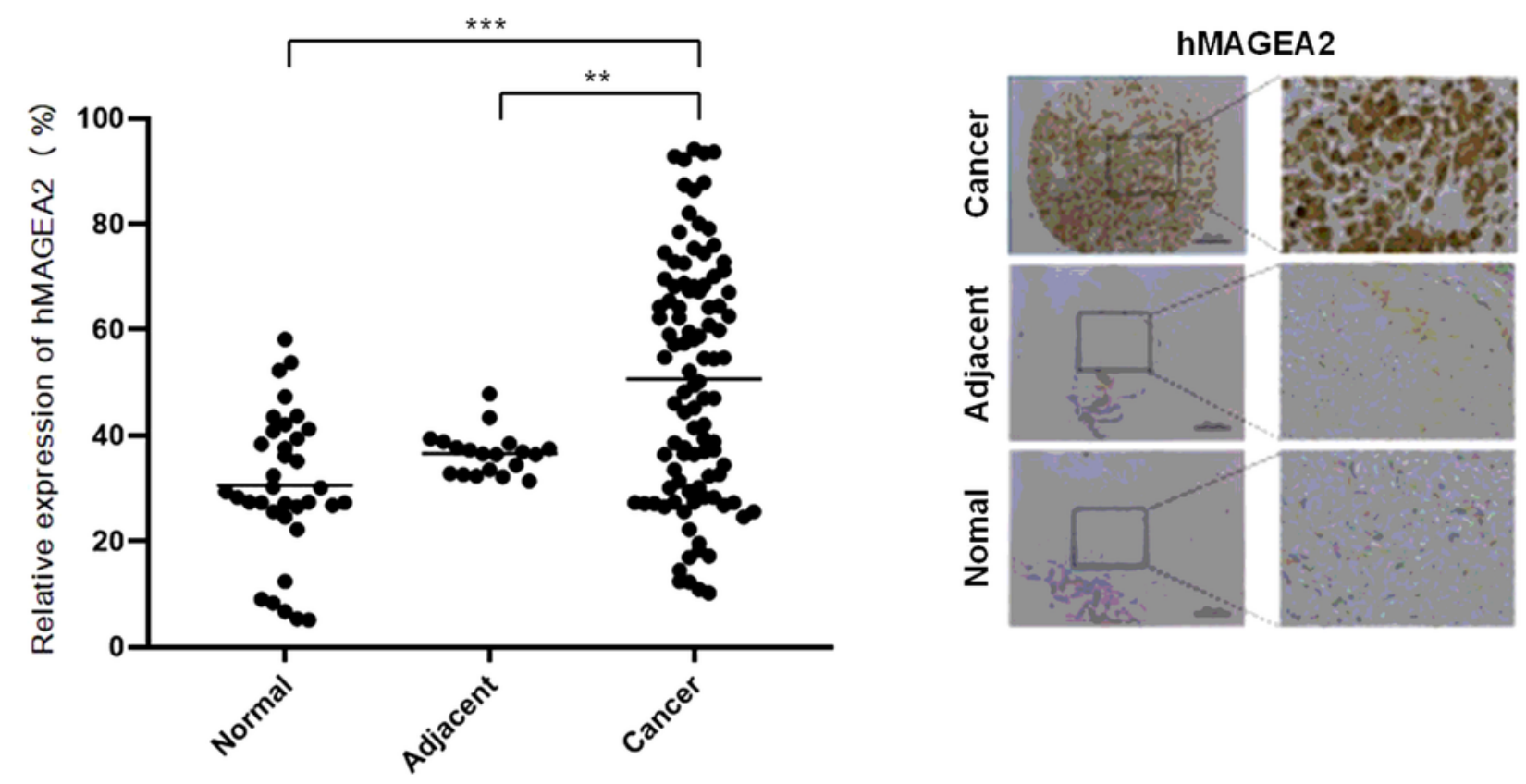

C

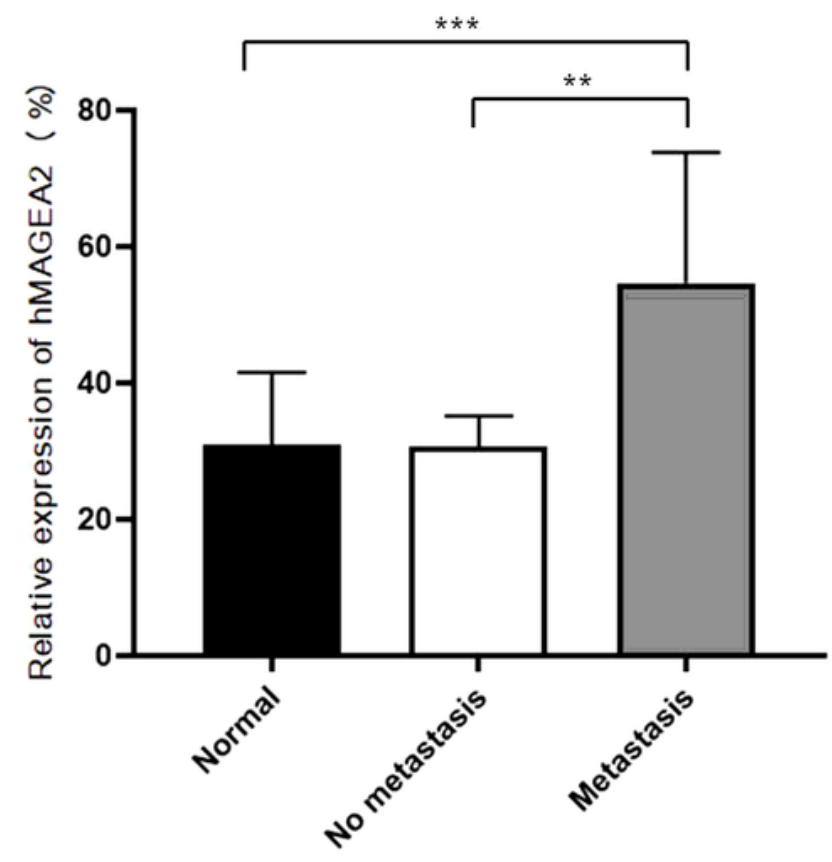

Figure 1

hMAGEA2 is highly expressed in human melanoma tissues. (A) Expression of hMAGEA2 in human melanoma tissues. (B) Immunostaining of hMAGEA2 in human melanoma tissues. (C) Expression of hMAGEA2 in melanoma with or without metastasis. Data are expressed as mean $\pm S D ; \star \star P<0.01, \star \star \star P<$ 0.001 , relative to control. Experiments were performed in triplicate. 
A

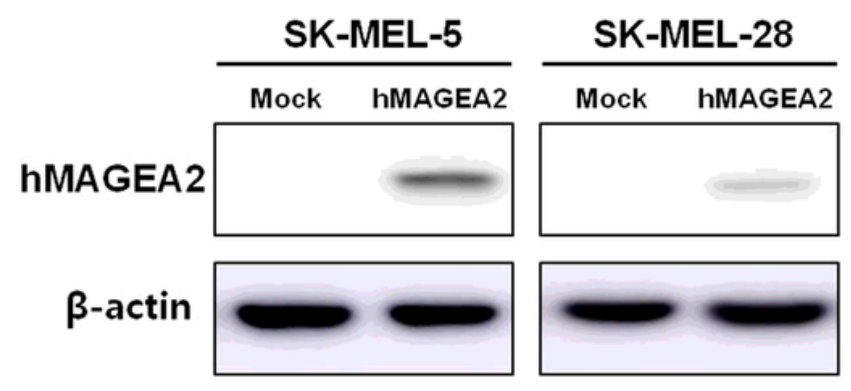

B
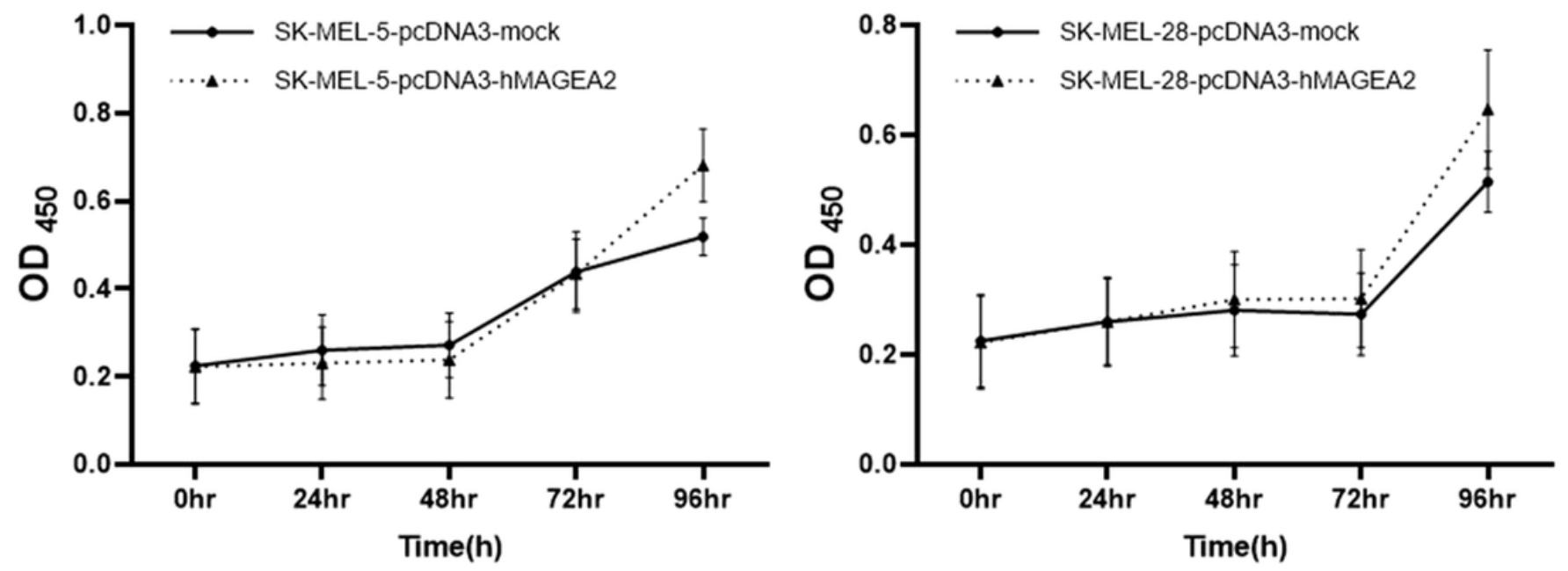

Figure 2

Overexpression of hMAGEA2 promoted melanoma cell proliferation. (A) Protein expression of stable hMAGEA2 overexpression in SK-MEL- 5 and SK-MEL-28 cell lines, $\beta$-actin was used as a loading control. (B) Proliferation was increased in melanoma cells that overexpress hMAGEA2 relative to control cells. Data are expressed as mean $\pm S D ; * \star P<0.01$, relative to control. 
A

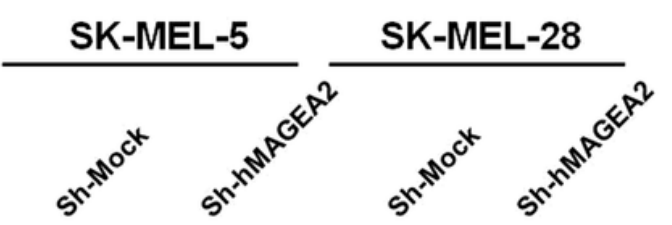

hMAGEA2
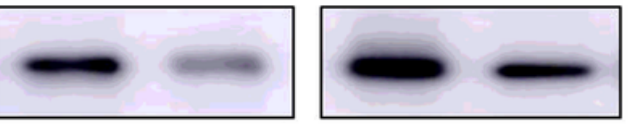

$\beta$-actin
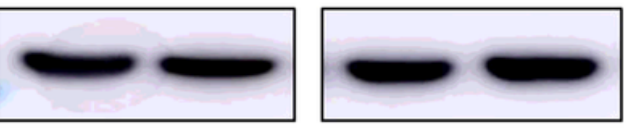

B
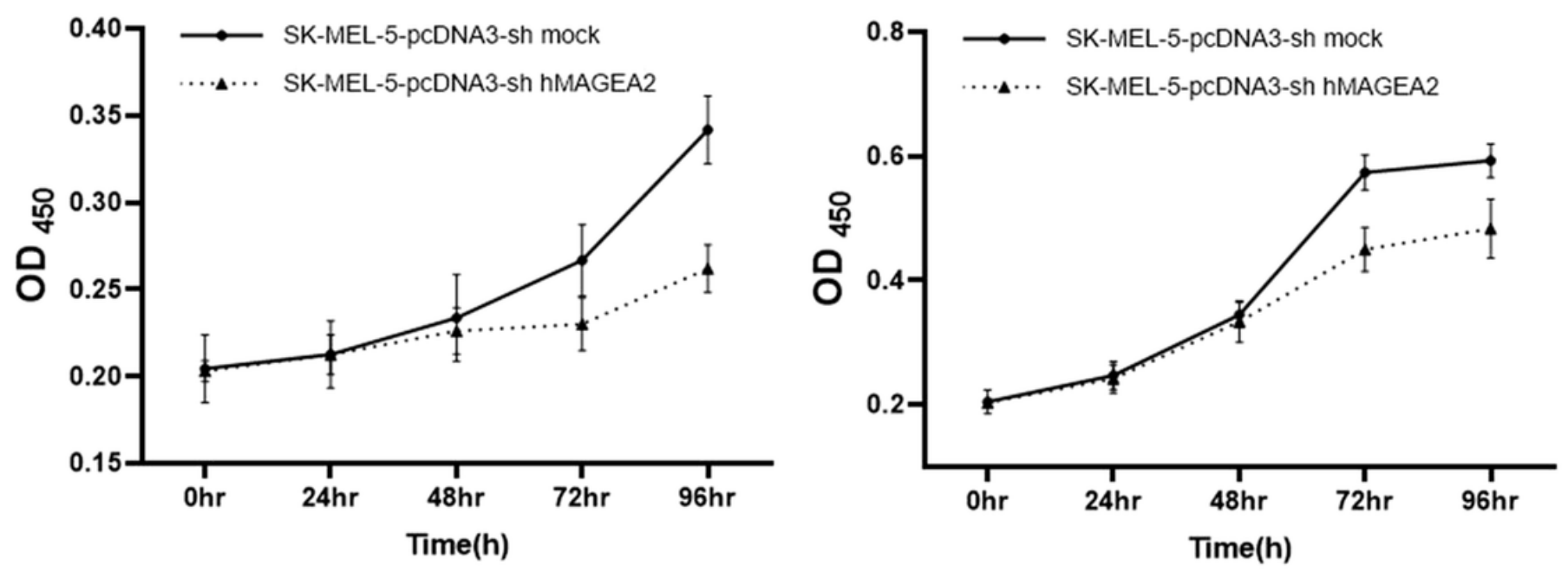

Figure 3

Knockdown of overexpressed hMAGEA2 in melanoma cells suppresses cell growth. (A) hMAGEA2 expression in SK-MEL-5 and SK-MEL-28 cell lines in the presence of mock or short hairpin RNA translation inhibitors. $\beta$-actin was used as a loading control. (B) Proliferation was suppressed in cells containing short hairpin RNAs specific for hMAGEA2 inhibition relative to cells containing nonspecific RNAs (mock) relative to control cells. Data are expressed as mean $\pm S D$; $* \star P<0.01$, relative to control. 
A

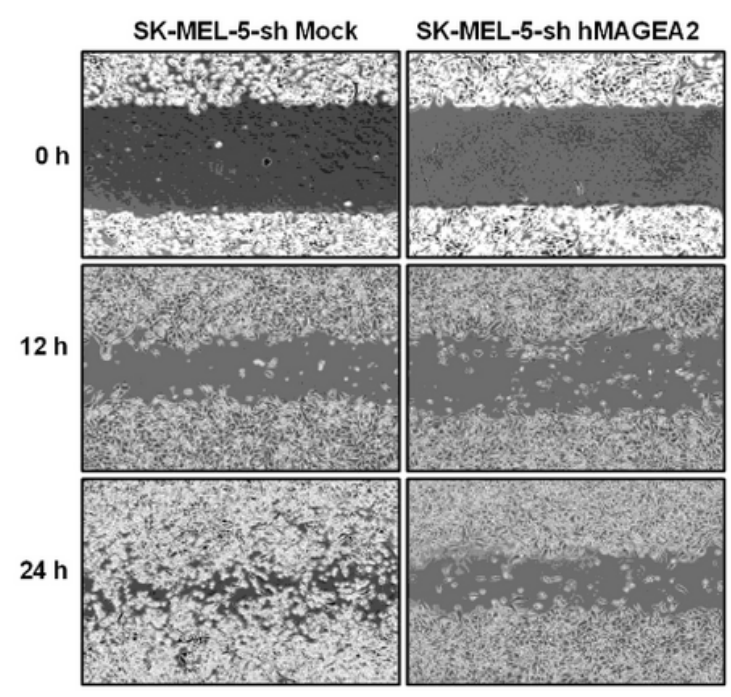

B

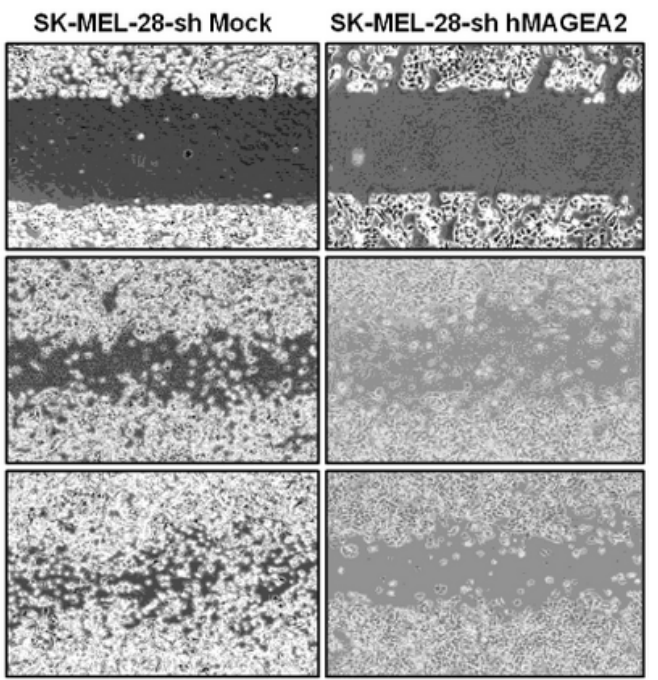

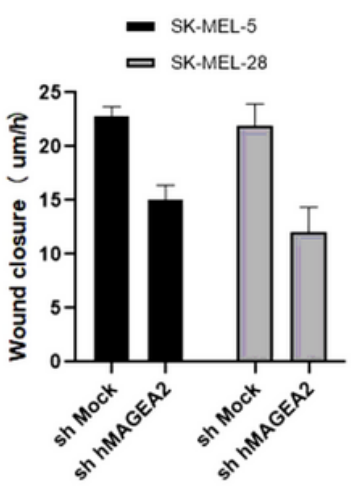

Figure 4

Knockdown of hMAGEA2 inhibits the motility of melanoma cells. (A) Cell motility of SK-MEL-5 sh-MOCK cells and SK-MEL-5 sh-hMAGEA2 cell lines was evaluated by wound-healing assays. (B) Cells were plated in complete medium at a confluent density and scratched with a micropipette tip. Average rates of wound closure were calculated from three independent experiments. Bar, $300 \mu \mathrm{m}$. Data are expressed as mean \pm SD; ${ }^{*} \mathrm{P}<0.05$.

A

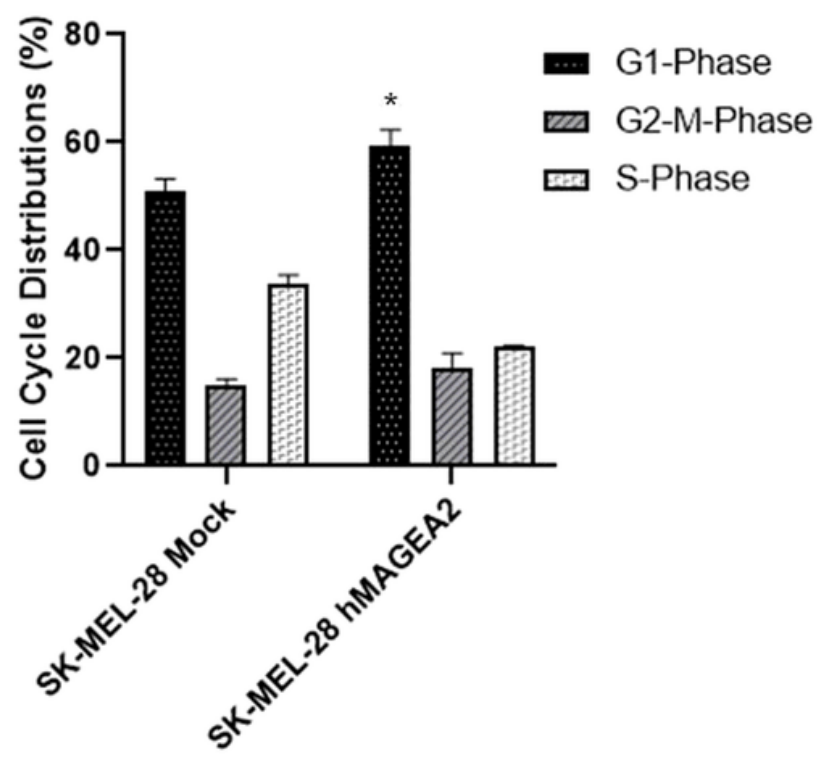

B

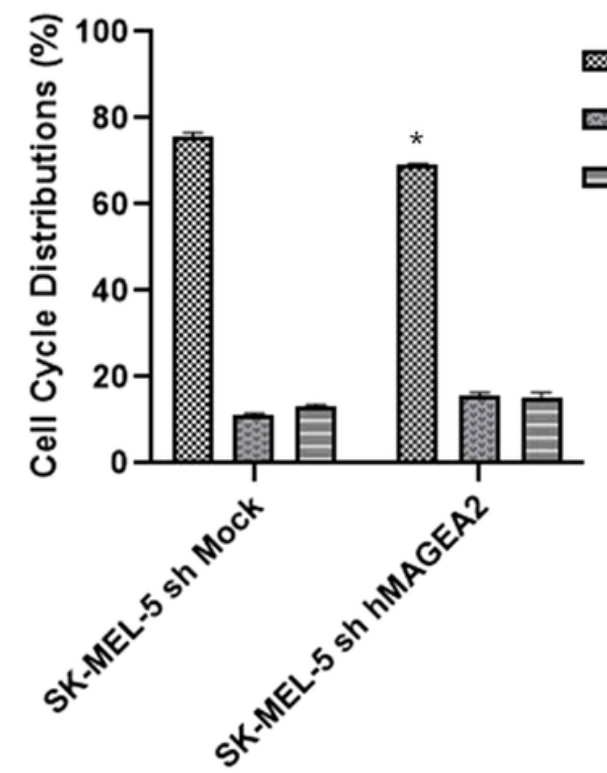


hMAGEA2 affects melanoma cells cell-cycle. (A) Percentage of cells in G1 phase in the hMAGEA2 Overexpression group was higher relative to the SK-MEL-28 sh-MOCK cells. (B) Knockdown of hMAGEA2 increases the percentage of cells in S phase in SK-MEL-5 cells. G2-M: percentage of cells in G2 or M phase. Data represent mean $\pm S D ; * P<0.05$.

A

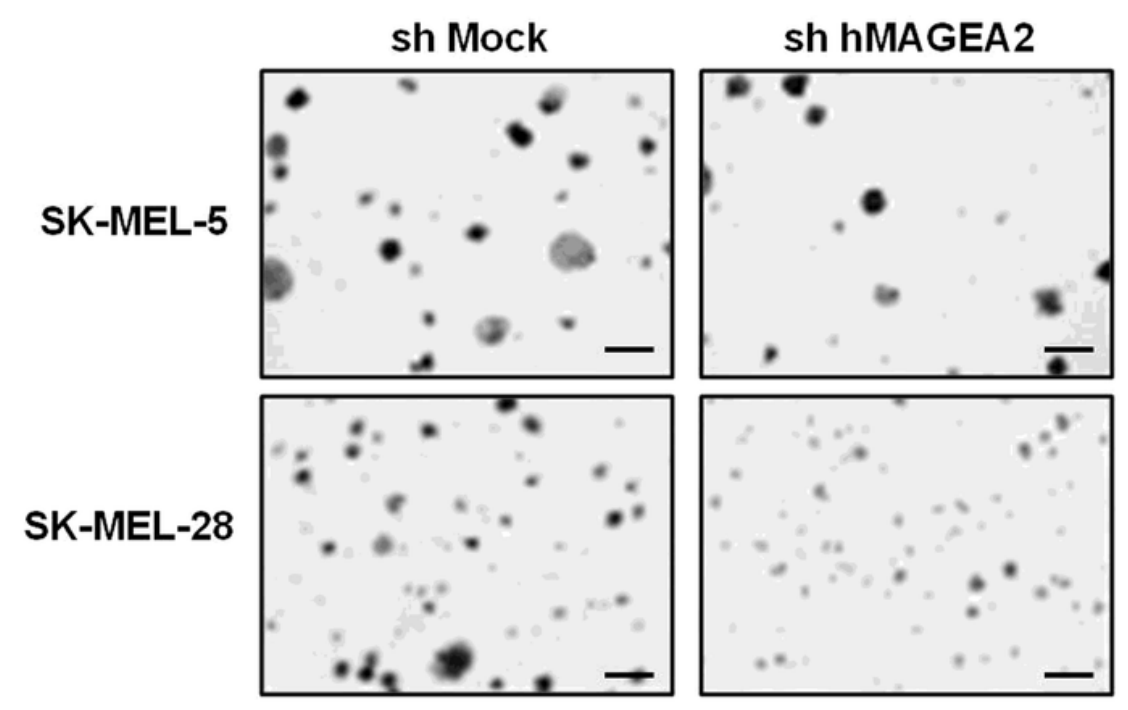

B

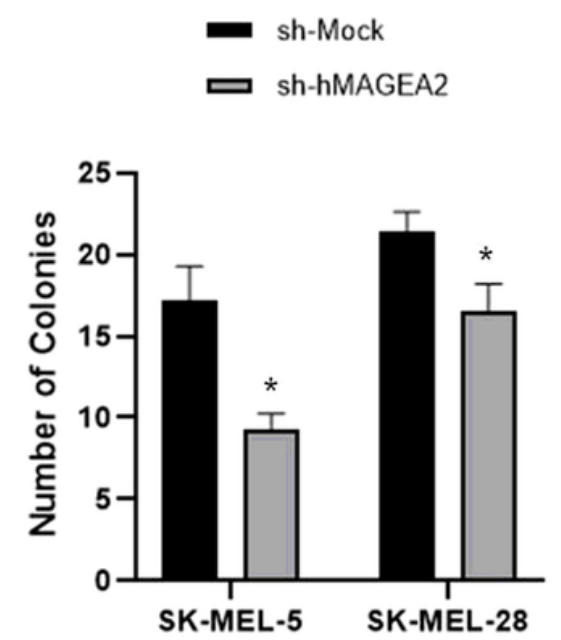

Figure 6

Knockdown of hMAGEA2 protein suppresses the anchorage independent growth of melanoma cells. (A) Images of colonies formed by the indicated cell lines transfected with the indicated knockdown vectors.

(B) Colony numbers in the indicated cell lines transfected with the indicated knockdown vectors. 
A
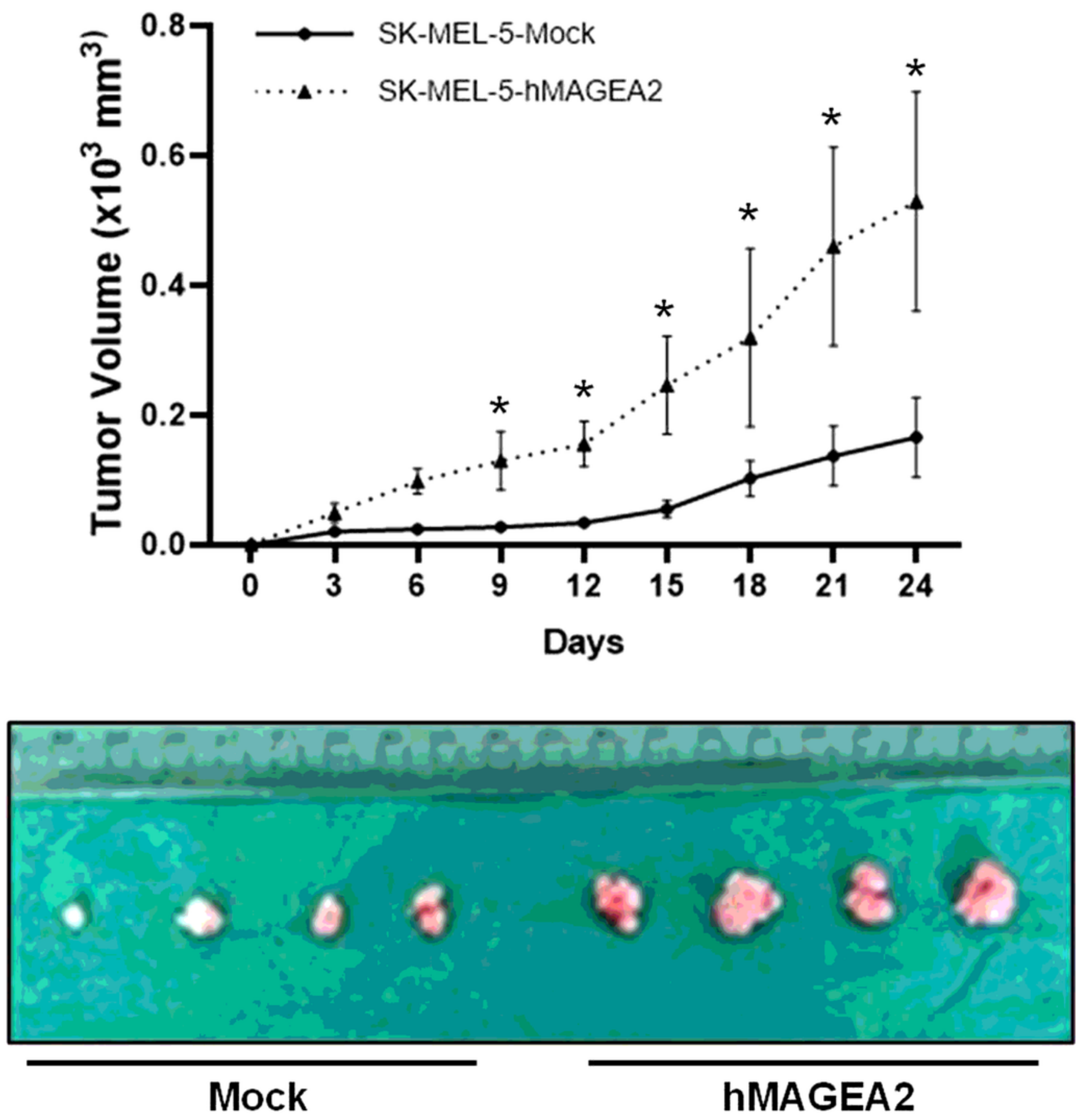

Figure 7

hMAGEA2 enhances xenograft tumor formation (A) Growth curve of xenograft tumor formation after injection of SK-MEL-5 cells that overexpress hMAGEA2 or do not overexpress hMAGEA2 into Balb/c nude mice. Data represent mean $\pm S D ; * P<0.05$. 